\title{
GEncode: Geometry-driven compression for general meshes
}

\author{
Thomas Lewiner ${ }^{1,2}, \quad$ Marcos Craizer ${ }^{1}, \quad$ Hélio Lopes $^{1}, \quad$ Sinésio Pesco ${ }^{1}, \quad$ Luiz Velho $^{3}$ And \\ ESDRAS MEDEIROS ${ }^{3}$ \\ ${ }^{1}$ Department of Mathematics — Pontifícia Universidade Católica — Rio de Janeiro — Brazil \\ ${ }^{2}$ Géométrica Project — INRIA - Sophia Antipolis — France \\ ${ }^{3}$ Visgraf Project - IMPA — Rio de Janeiro - Brazil \\ \{tomlew, craizer, lopes, sinesio\}@mat.puc--rio.br. \{lvelho, esdras\}@visgraf.impa.br.
}

\begin{abstract}
Performances of actual mesh compression algorithms vary significantly depending on the type of model it encodes. These methods rely on prior assumptions on the mesh to be efficient, such as regular connectivity, simple topology and similarity between its elements. However, these priors are implicit in usual schemes, harming their suitability for specific models. In particular, connectivity-driven schemes are difficult to generalise to higher dimensions and to handle topological singularities. GEncode is a new single-rate, geometry-driven compression scheme where prior knowledge of the mesh is plugged into the coder in an explicit manner. It encodes meshes of arbitrary dimension without topological restrictions, but can incorporate topological properties, such as manifoldness, to improve the compression ratio. Prior knowledge of the geometry is taken as an input of the algorithm, represented by a function of the local geometry. This suits particularly well for scanned and remeshed models, where exact geometric priors are available. Compression results surfaces and volumes are competitive with existing schemes.
\end{abstract}

Keywords: Mesh Compression. Geometry-driven techniques. Arbitrary Meshes. Arbitrary Dimension.

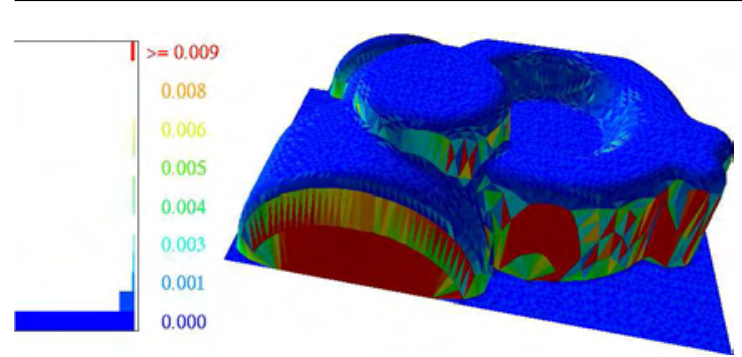

1(a): geometric criterion
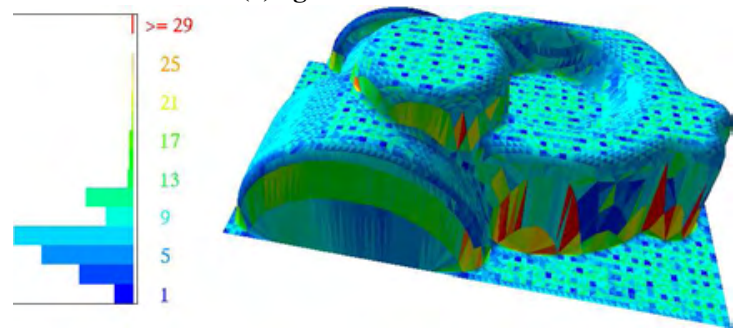

1(c): max identifier

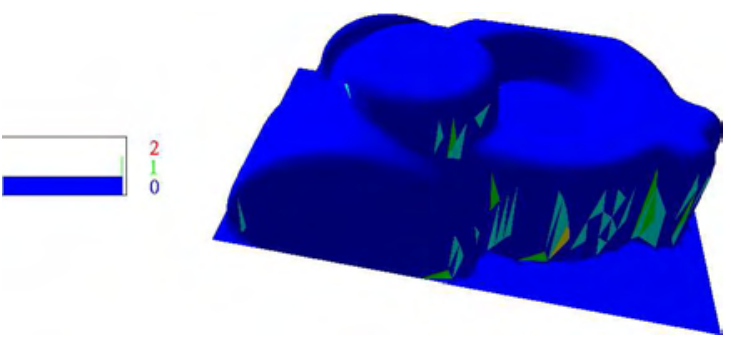

1(b): geometric range

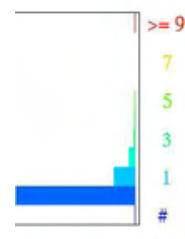

1(d): apex identifier

Figure 1: GEncode compression: once the geometry is decoded, the decoder attaches triangles to edges of the front by identifying its apex $w$ : A list of candidates is computed from an encoded geometric range and ordered according to a geometric criterion (here the distance from $w$ to the edge midpoint). Then $w$ is identified by its position in the list.

\section{Introduction}

Preprint MAT. 02/06, communicated on March $17^{\text {th }}, 2006$ to the Department of Mathematics, Pontifícia Universidade Católica - Rio de Janeiro, Brazil.
Computer Graphics developments handle each time bigger meshes, using processes of increasing complexity. Compression algorithms followed these developments by 
improving the compression ratio, enlarging the range of models that can be encoded, simplifying their implementation and increasing execution performances. However, they are still not fully adapted to the wide variety of models and applications of Computer Graphics: scans in artistic and archaeological modelling, isosurfaces for medical and mathematical visualisation, re-meshed models for reverse engineering, finite-element meshes for simulation, highdimensional meshes for solid representation, meshes with high co-dimension for non-linear optimisation, among others. Actually, the performances of state-of-the-art compression algorithms highly depend on the nature of the model. We will focus here on compression schemes adapted to the priors of specific applications, in particular for the most time-consuming mesh generation algorithms: reconstruction and the re-meshing.

Geometry-driven compression. Meshes are usually described by their geometry (the coordinates of its vertices) and their connectivity (the combinatorial elements that interpolate these vertices, usually triangles or simplices). This composite nature leads to classify compression algorithms between: on one hand connectivity-driven ones, when the connectivity is coded separately and the geometry is partially deduced from it, and on the other side geometrydriven ones, when the geometry is coded separately and the connectivity is coded using the geometry. The efficiency of connectivity-driven algorithms usually relies on a regular connectivity, whereas the newer trend of geometrydriven methods are expected to perform better on geometrical meshes, such as reconstructed or re-meshed models. This work proposes a new geometry-driven method, which encodes arbitrary meshes in arbitrary dimension, and compares nicely to connectivity-driven methods for surfaces (Figure1) and for volumes (Figure 11).

Related works. The first mesh-compression algorithms were connectivity-driven, in the sense that the geometry encoding depends on the connectivity encoding rules. Among those, the Edgebreaker [33, 29, 27, 23] performs well on generic mesh, with guaranteed practical worst-case close to the theoretical optimum [19]. On the other side, Valence Coding [35, 9, 18] has a theoretical asymptotic compression ratio close to the optimum [1]. It has been widely extended since the original work, and performs very well in practise, especially on meshes with a regular connectivity. Some singularities of the mesh can further be handled by specific algorithm, in particular for the non-manifold case [13, 30].

These connectivity-driven approaches can be extended to higher dimension, but the complexity of the codes increases dramatically. Even for tetrahedral meshes, the extensions of the surface approaches [14, 32, 17] are delicate.

As an intermediate towards geometry-driven ap- proaches, some connectivity-driven schemes use the previously coded geometry to predict the connectivity [20, 21, 11, 18]. Each of these algorithms uses a different prior on the geometric regularity of the mesh.

On the contrary, geometry-driven approaches introduced by [12] handle gracefully complex connectivity. Still, the compression ratios of the geometry are not yet optimal, since these schemes are quite new to the community. However, for the case of isosurfaces, specific compression schemes [34, 22, 24] outperform any connectivity-driven approach.

Contributions. This work proposes a new geometrydriven scheme called GEncode, which works for meshes of arbitrary topology and dimension embedded in spaces of arbitrary dimension. To our knowledge, GEncode is the first compression method that works at that level of generality and still compares nicely to state-of-the-art compression methods for triangulated surfaces and volumes. As opposed to [12], GEncode is single rate, but copes with general meshes, and shows better compression ratios: For surface, the resulting compression ratios are competitive with the Edgebreaker with the parallelogram prediction, and for volumes it is highly competitive with Grow\&Fold [32] and with streaming compression [16].

Aside from its generality, GEncode treats the priors of the mesh as an input, and can therefore easily adapt to specific classes of meshes. These priors include on one side global topological properties such as manifoldness, the presence of boundary and eventually the degree of the facets, and on the other side local geometrical properties represented by a scalar function of the vertices of a facet. For example, a common prior for usual Computer Graphics models assumes that the mesh is a triangulated manifold without boundary, and that the triangles maximise their circumradius or their aspect ratio. In particular, if the mesh can be reconstructed from its vertices with a geometric prior, the GEncode connectivity encoding with that prior leads to a zero entropy code.

This work is an extended version of [25] and part of Thomas Lewiner's Ph.D. [26]. It describes GEncode at its high level of generality, investigates different geometric priors and separates the geometric range definition from the geometric prior in order to reduce the constraints on the geometric function defining the prior. Moreover, the authors are grateful to the referees, since they motivated tests on tetrahedral meshes, where GEncode turned out to be particularly competitive.

Overview. This work is organised as follow. section 2 General Meshes recalls the basic notions of meshes, expressed in arbitrary dimension. Then section 3 Independent Encoding of the Geometry introduces the two methods we considered for compressing the geometry, and how we synthe- 


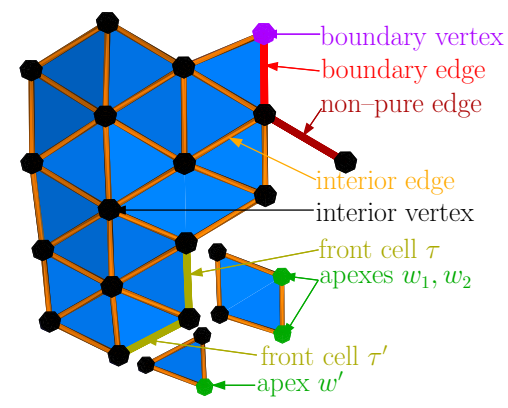

Figure 2: Cell complex elements topology and cell attachment operation.

sised them. The main part of GEncode is introduced at section 4 Connectivity Encoding: Geometric Range and Apex Identifiers, followed in section 5 Geometric Priors by a discussion on priors that can be plugged into the algorithm to compress efficiently usual models. Finally, section 6 Results provides some results and comparisons with state-of-theart methods on common models, and compares them with the Edgebreaker for surfaces, and with Grow\&Fold and streaming methods for volumes.

\section{General Meshes}

This section introduces the basic definitions that are used in this work, especially the notion of convex cell complexes [15]. This notion is introduced formally, but corresponds to the usual meshes used in Computer Graphics, and the reader can think of this notion as a generalisation of triangulated surfaces. They can be constructed in an incremental manner by the single operation of cell attachment. This construction is usually referred as advancing front, and entails most of the mesh decoding algorithms.

Convex cells. A convex cell $\sigma$ in $\mathbb{R}^{p}$ is a non-empty compact subset of $\mathbb{R}^{n}$ which is the solution set of a finite number of equations $f_{i}(\mathbf{x})=0$ and inequalities $g_{i}(\mathbf{x}) \geqslant 0$, where $f_{i}$ and $g_{i}$ are affine functions of the form $\left(x_{1}, x_{2}, \ldots, x_{p}\right) \mapsto$ $\lambda_{0}+\lambda_{1} x_{1}+\lambda_{2} x_{2}+\ldots+\lambda_{p} x_{p}$.

A cell $\sigma^{d}$ has dimension $d$ if it contains $d+1$ affine independent points but no more. A subcell $\tau$ of $\sigma$ is a cell obtained by changing some of the inequalities $g_{i}(x) \geqslant 0$ to equalities. We will say that $\sigma$ is incident to $\tau$. The collection of all the subcells of $\sigma^{d}$ of dimension $d-1$ is denoted $\partial \sigma$.

Points, line segments, triangles, quadrangles, tetrahedræ, cubes are examples of convex cells. Among these convex cells are the simplices, which generalise the notion of line segment, triangle and tetrahedron: A $d$-simplex is the convex hull of $(d+1)$ affine independent points in the space.

Convex cell complex. A convex cell complex $K$ is a coherent collection of distinct convex cells, where coherence means that the collection contains the subcells of each cell and the intersection of any two cells. A convex cell complex $K$ is pure of dimension $n$ if every cell in $K$ is of dimension $n$ or is a subcell of a cell of dimension $n$ belonging to $K$. A facet of a pure $n$-complex $K$ is a cell of $K$ of dimension $n$.

The vertices of a cell are its subcells of dimension 0 . The geometry of a complex usually refers to the coordinates of its vertices, while its connectivity refers to the incidence of higher dimensional cells on these vertices. Observe that a cell is uniquely determined by its vertices.

Cell attachment. An $n$-cell $\sigma$ can be attached to a complex $K$ by identifying a collection of its subcells $\left\{\tau_{1}, \cdots, \tau_{k}\right\}$ with some of the cells of $K$, preserving its nature of cell complex.

If one of the cells $\tau$ of $K$ is of dimension $n-1$, this cell attachment can be considered as the attachment of $\sigma$ onto $\tau$, and we will write $\sigma=\tau \star\left\{w_{1}, \ldots, w_{m}\right\}$, where the vertices $\left\{w_{1}, \ldots, w_{m}\right\}$ are those of $\sigma$ not subcells of $\tau$ (Figure 2). These vertices are called the apexes of the cell attachment. If $\sigma$ is a simplex, there is only one apex $(m=1)$.

Manifolds. Among these convex cell complexes, the class of combinatorial manifolds is the most widely used. A combinatorial $n$-manifold $\mathcal{M}$ is a pure complex of dimension $n$ where for each vertex $v$, the union of each open simplex containing $v$ is homeomorphic to the open $n$-ball $\mathbb{B}^{n}$ or the intersection of $\mathbb{B}^{n}$ with a closed half-space. This implies that each $(n-1)$-cell is a subcell of either one or two $n-$ cells. The set of $(n-1)$-cells subcells of only one $n$-cell is called the boundary of $\mathcal{M}$ (Figure 2).

\section{Independent Encoding of the Geometry}

GEncode is a pure geometry-driven scheme, and the coordinates of the vertices of the mesh are thus encoded separately before the connectivity compression. We considered two geometry coding techniques, described in [12] and in [8], and propose a synthesis of them. This synthesis has similar compression ratios as both [12] and [8], but emphasises their strong points and could be the basis for further improvements on this part of the coding. In our experiments, the proposed synthesis is generally more efficient on small models (below 3000 vertices) or on the threedimensional meshes we tested (Figure 11), whereas [12] gets the best results for larger models.

Space partition encoding. The coordinates of all the vertices are encoded globally as a space partition tree. This kind of techniques works for vertices with an arbitrary number of coordinates, allowing encoding meshes of arbitrary co-dimension. In particular in [12] and [8], the space is divided with a particular binary space partition where each separator is perpendicular to the axis, as an octree for dimension 3: the axis alternates from one level to the next one $\left(X, Y, Z, X, Y \ldots\right.$ in $\left.\mathbb{R}^{3}\right)$, and each part is subdivided in two equal sub-parts, as on Figs. 3, 4 and 5, The subdivision 
is performed until each part contains only one vertex. We will now compare and synthesise these techniques.
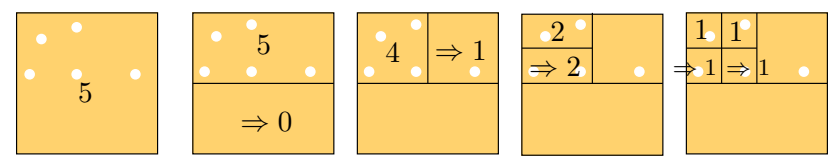

Figure 3: The geometry encoder of $[12]$ codes first 5 as a standard 32 bits number, then 5 on $\left[\log _{2}(6)\right]$ bits, and then 4 on $\left\lceil\log _{2}(6)\right\rceil$ bits. The right vertex relative position is then coded. Then 2 is coded on $\left\lceil\log _{2}(5)\right\rceil$ bits, and both 1 on $\left\lceil\log _{2}(3)\right\rceil$ bits. The position of the last vertices is coded.

Lower nodes efficient. In [12], each node of the space partition is encoded by the number of vertices $\# v_{l}$ its left children contains. The number of vertices of the other node $\# v_{r}$ is simply deduced by difference from the number of the vertices of the parent's node $\# v_{f}$ which is known from the recursion. This technique wastes many bits at the beginning of the encoding: the number of nodes must be encoded on $\left\lceil\log _{2}\left(\# v_{f}+1\right)\right\rceil$ bits, since the number of node of the left child can be $\left\{0, \ldots, \# v_{f}\right\}$ (except the first node which is on 32 bits by convention), as for the example of Figure 3 At the end, when there is only one vertex per node, it is encoded with 1 bit per level, which is optimal.
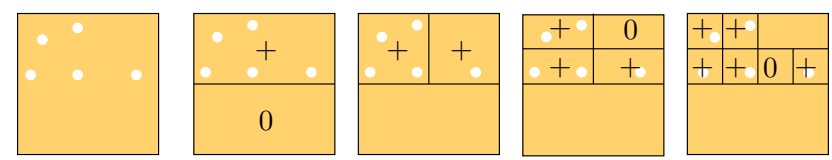

Figure 4: The geometry encoder of $[\overline{8} \mid$ codes the following sequence: $+0,++,++, 0+,++,++, 0+$. Then follow $0+$ and +0 to reach the desired number of bits.

Higher nodes efficient. In [8], each node is encoded by one out of 3 symbols: ++ if both children contain at least one vertex, +0 if only the left child contains a vertex, and $0+$ if only the right one contains a vertex, as for the example of Figure 4. Note that at least one child must contain a vertex, since the parent did. The encoding stops at a predefined level. This method spends more bits at the end of the encoding, since the decoder does not know when there is only one vertex in a node. Therefore, the encoder sends $\log _{2}(3)$ bit for each level, which is greater than the 1 bit of [12] for the last part.

Synthesis proposal. The goal of this synthesis is to take the best part of both. First, it encodes each node by one of 6 symbols: ++ if both children contains more than one vertex, +1 and $1+$ if one child contains more than one vertex, and the other only one, 11 if they both contain only one vertex, and +0 and $0+$ if one child contains more than one vertex, and the other child is empty, as for the example of Figure 5. With this encoding, the encoder
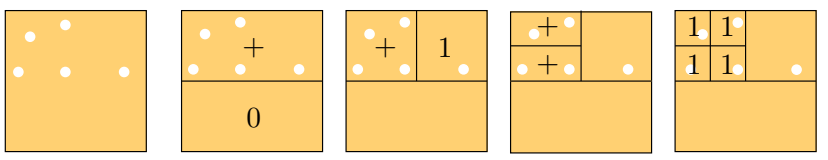

Figure 5: Synthesis: the encoder codes the following sequence: $+0,+1$ and the right vertex relative position is then coded, and then $++, 11,11$. The position of the remaining vertices is then coded.

detects when there is only one vertex in a node, and then uses the technique of [12]. The coder further benefits from the different probability of the symbols. Moreover, these probabilities are used differently depending on the level of the node to encode: nodes closer to the root are more frequently of type ++ , whereas these are rare when going closer to the leaves.

\section{Connectivity Encoding: Geometric Range and Apex Identifiers}

The main contribution of GEncode is its connectivity coding (Algs. 11 gencode and 2 , gdecode). As a generic geometry-driven compression scheme, the geometry is known before the connectivity is decoded, either because it is already available as a point cloud, or because it has been decoded by a method like the one we presented above. The connectivity decoding then works similarly to greedy advancing front reconstruction algorithms such as [6, 28, 31], but the best match used by the reconstruction algorithm is continuously corrected by the encoded stream. Therefore, for meshes that can be reconstructed with a greedy strategy, GEncode can achieve a zero entropy message for the connectivity.

Coding Principle. The algorithm encodes an initial $n-$ cell and then works as an advancing front triangulation, maintaining an ordered queue of $(n-1)$-cells and attaching at each step a cell $\tau \star\left\{w_{1}, \ldots, w_{m}\right\}$ to $\tau$, the cell of the front with the highest priority, or removing $\tau$ from the front, for example when $\tau$ is on the boundary of the final mesh. When an $n$-cell is attached, its $(n-1)$-faces are added to the front. The compression of a connected component ends when the front is empty. The difference with greedy reconstruction algorithms is that vertices $w_{j}$ are not always the ones that minimise a given geometric criterion $\mathcal{G}\left(\tau, w_{j}\right)$, such as the circumradius of $\tau \star w_{j}$ used by [6]. Such criteria will be discussed in section 5 Geometric Priors. To identify the apex $w_{j}$, we actually encode its position in a list of candidates, generated by a geometric range $\left[\mathcal{R}_{\min }, \mathcal{R}_{\max }\right]$ and ordered by geometric criterion $\mathcal{G}$.

Geometric Range. The list of candidates for $w$ could be the list of all vertices, but that would allow $O(\#$ verts $)$ choices, which is too expensive to encode. In order to reduce the size of that list and the time used to compute it, 


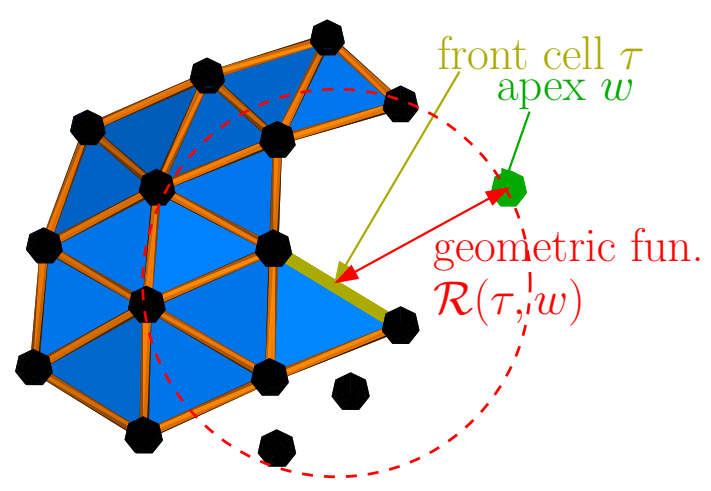

6(a): Coder side: geometric function $\mathcal{R}(\tau, w)=$

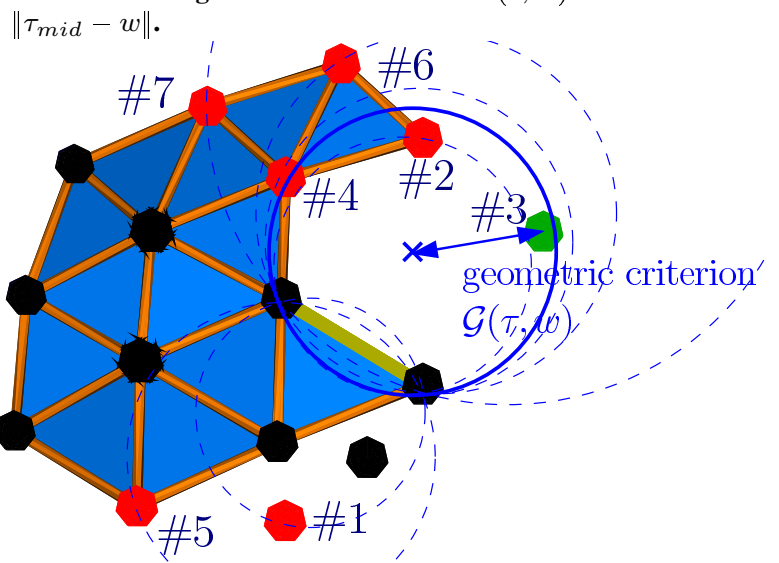

6(c): Both sides: apex candidatés are ordered by the geometric prior $\mathcal{G}\left(\tau, v_{i}\right)=\bar{\rho}\left(\tau \star v_{i}\right)$.

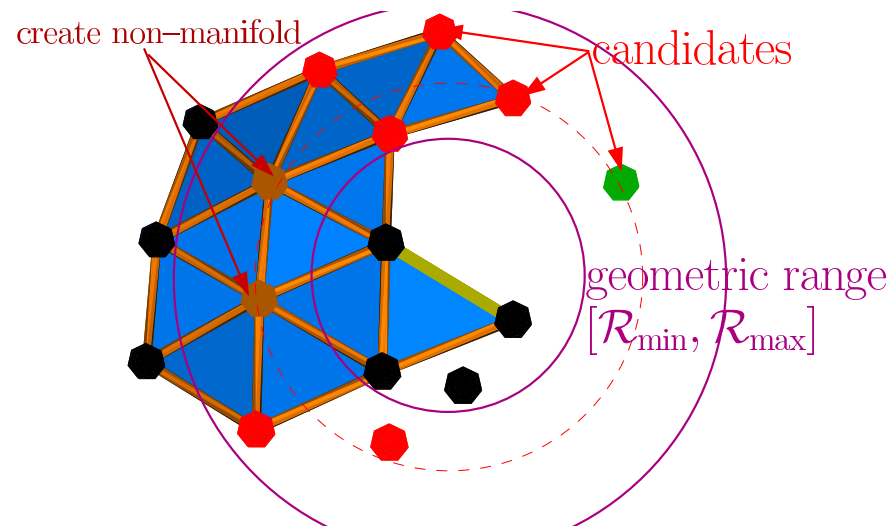

6(b): Both sides: geometric range $\mathcal{R}_{\min } \leqslant \mathcal{R}(\tau, w)<\mathcal{R}_{\max }$.

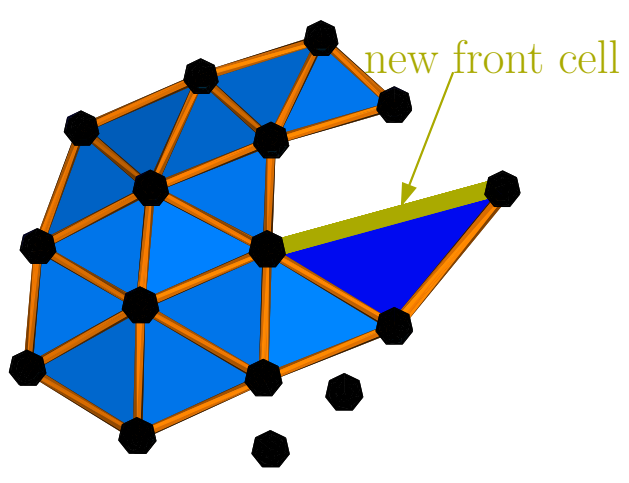

6(d): Decoder side: the decoded apex identifier \#3 defines the cell attachment $\tau \star w$.

Figure 6: GEncode principle: the geometric function (a) is encoded. Its quantisation restricts the apex candidates, which are then filtered by the topological priors (b). The candidates are ordered (c) and the encoder transmits the candidate number in the list (here \#3), which allows the decoder to perform the cell attachment (d) and continue on the next front cell.

a geometric function $\mathcal{R}(\tau, w)$ is transmitted (Figure 6(a)). However, the quantisation process encodes $\mathcal{R}(\tau, w)$ as one of the predefined ranges: $\mathcal{R}(\tau, w) \in\left[\mathcal{R}_{\min }, \mathcal{R}_{\max }\right]$. The list of candidates will then be the vertices $v_{i}$ such that $\mathcal{R}\left(\tau, v_{i}\right)$ belongs to $\left[\mathcal{R}_{\text {min }}, \mathcal{R}_{\text {max }}\right]$ (Figure 6(b)).

The definition of the geometric function $\mathcal{R}$ must be effective for identifying the candidate vertices. In particular, for simple geometric priors $\mathcal{G}$ on the mesh, we choose $\mathcal{R}=\mathcal{G}$. Moreover, the binary space partition of the geometric encoding is used to accelerate the localisation of the candidates.

Apex Identifiers. Once the list of apex candidates $\left\{v_{i}\right\}$ is generated, it is ordered by the geometric prior $\mathcal{G}\left(\tau, v_{i}\right)$. For example on Figure 6(c) the best candidate according to $\mathcal{G}$ gets identifier 1 , the second best match receives identifier 2. The original apex gets identifier 3 , and this number is transmitted to the decoder, which can then perform the right cell attachment (Figure 6(d)). This ordering gives a higher probability to the lower identifiers and thus reduces the entropy.

Non-simplicial meshes. If the mesh is not simplicial, a third number needs to be encoded: the number $m$ of apexes for the cell attachment $\tau \star\left\{w_{1}, \ldots, w_{m}\right\}$ (line 8 of Alg. 11 gencode). Moreover, there is one range per apex $w_{j}$, but only the smallest and the biggest ranges of the $w_{j}$ are actually encoded. In that case, we know that the second and following apexes are restricted to be on the affine hyperplane defined by $\tau \star w_{1}$. This reduces the candidate list, which improves the entropy of the apex identifiers stream.

Quantisation trade-off. This scheme actually encodes the apex by two means: the geometric range and the apex identifier. The quantisation of the geometric function is thus a trade-off: On one hand, if the quantisation is rough, there will be many candidates, which requires more time to generate the list and an expensive encoding of the position of $w_{j}$ in the list of the candidates, as show the histograms 


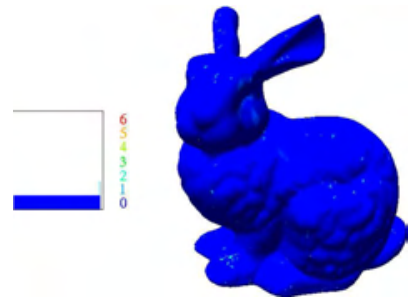

7(a): range: roughly quantised (low entropy).

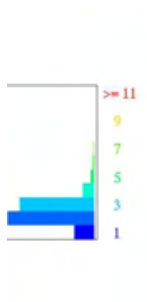

7(b): number of candidates (high entropy).
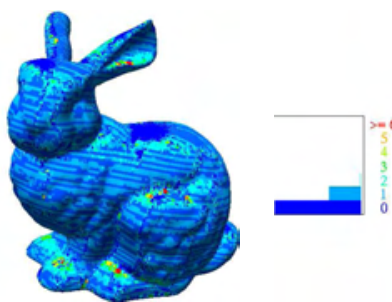

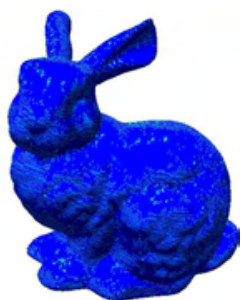

7(c): range: precisely quantised (high entropy).
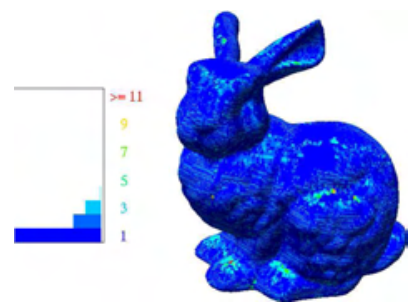

7(d): number of candidates (low entropy).

Figure 7: Quantisation trade-off: rough quantisation of $\mathcal{R}$ leads to lower entropy for the range, but more candidates, thus higher entropy for the apex identifier.

of Figure 7 On the other hand, if the quantisation is too refined, the quantised geometric range will be expensive to encode.

Topological Priors. GEncode compresses pure meshes of arbitrary dimension, embedded in any co-dimensional space, orientable or not, with any kind of topology. If the mesh contains more than one connected component, the algorithms are applied separately on each of them. Further

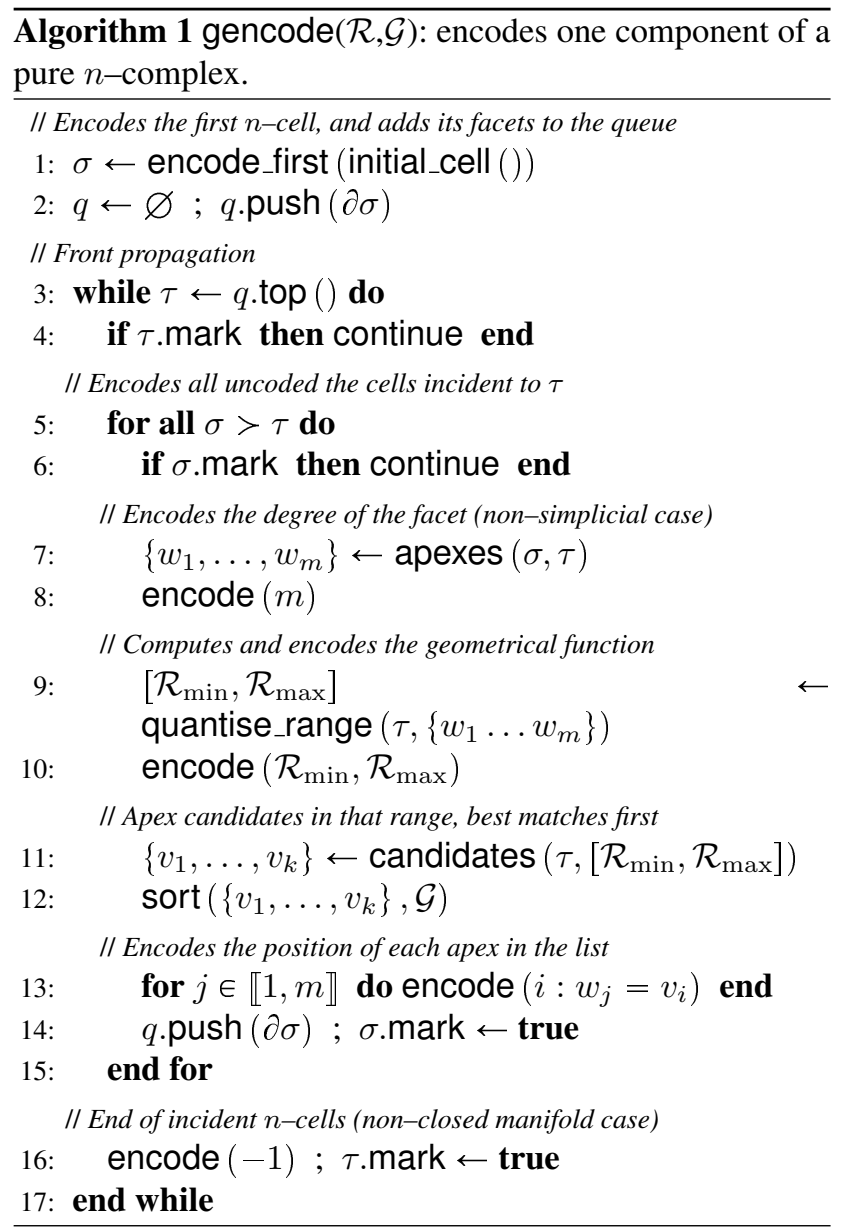

information on the mesh will then improve the compression ratio: We already saw that if the mesh is simplicial, the encoding of the face degree (parameter $m$ line 8 of Alg. 1. gencode) can be avoided. Even if this is not known to the coder, the sequences of $m$ will be constant, and thus have entropy zero.

Computer Graphics meshes are usually manifolds. In that case, GEncode is optimised in two ways: firstly, by removing from the list the candidates that would create a nonmanifold object, i.e. the vertices that are not on the boundary of the reconstructed mesh (Figure 6(b)). Secondly, an $(n-1)-$ cell will be processed at most once. Therefore, the $m=-1$ code of line 16 of Alg. 1. gencode only serves as

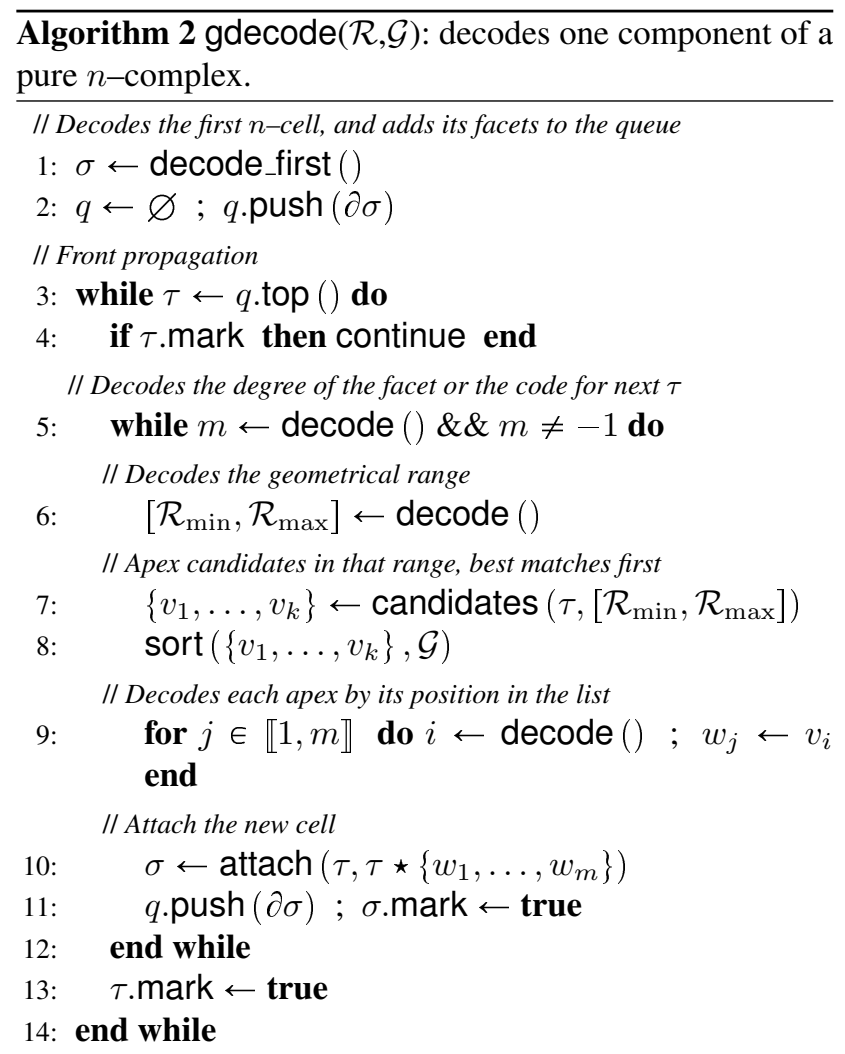


a marker for boundary cells, reducing its range to $0 /-1$ and thus improving the entropy of that code. If the complex is a simplicial manifold without boundary, the encoding of $m$ can be omitted for both the number of apexes and the boundary marker (lines 8 and 16 of Alg. 1 gencode).

Non-pure complexes. The above algorithms can be extended to cope with more general topology. If the $n-$ complex $K$ is not pure, as the one of Figure 2, we first encode it as if it were a pure complex. The uncoded cells will then be the non-pure elements of $K$. They form a complex $K^{\prime}$ of dimension lower than $n$. We then encode $K^{\prime}$ as above and continue recursively. This involves at most $n-1$ calls to Alg. 11 gencode or Alg. 2, gdecode, which maintains the linear complexity of these algorithms (considering that the localisation of the candidates is constant).

Guarantees. Given a convex cell complex $K$, the GEncode compression encodes a sequence of cell attachments, starting from an empty cell, and ending at $K$. The decompression reconstructs $K$ by this sequence, and identifies each cell attachment uniquely by the front cell, the number of apexes and their identifiers. The definition of a convex cell complex implies that this is enough to define the geometric realisation of the attached cell $|\sigma|$. However, there can be more than one combinatorial description of $|\sigma|$ if two $p$-cells of the boundary of $\sigma$ are aligned (contained in a $p$-affine plane). If this degenerated case does not occur in $K$, GEncode is then guaranteed to reconstruct $K$. If it occurs, the model can be either perturbed, or Algs. 1 gencode and 2 gdecode can be modified to encode the combinatorial structure of degenerated cells.

\section{Geometric Priors}

GEncode relies on two complementary geometric functions: the geometric range $\left[\mathcal{R}_{\min }, \mathcal{R}_{\max }\right]$ and the geometric prior $\mathcal{G}$ that orders the apexes by their probability to complete the front cell. In a rough sense, $\mathcal{R}$ encodes the highest bits of the apexes, which are usually the same as the highest bits of the front cell $\tau$, and $\mathcal{G}$ predicts the best apex in that range.

We will consider here two uses for the geometric function $\mathcal{R}$ : either to encode a specific geometric property of the mesh or to localise the apexes. In the first case, it should be equal to $\mathcal{G}$ (as in [25]). In the second case, its simplest expression would be the distance to the barycentre of the front cell $\tau$. We will thus focus now on $\mathcal{G}$.

Geometric criterion. The geometric criterion $\mathcal{G}(\tau, w)$ is an arbitrary real valued function that should be minimal with high probability when the cell $\tau \star w$ is a cell of the mesh. This criterion uses the local geometry of $\tau \star w$, and may take into account the decoded mesh, although we will not use this feature here. The more the criterion fits to the coded mesh, the better the compression ratio, since the parameter $i$ to encode on line 13 of Alg. 11 gencode will be equal or close to 0 with high probability, reducing the entropy of these codes.

Closest point criteria. The simplest geometric criterion is the distance to the barycentre of $\tau: \mathcal{G}_{d}(\tau, w)=$ $\|w-\operatorname{bary}(\tau)\|$. In that case, the geometric function $\mathcal{R}$ can be normalised by the volume (length) of $\tau$ quantised on $\left[0, \infty\right.$ [ with an exponential function: $\mathcal{R}_{d}(\tau, w)=$ $\left\lceil\log _{2}\left(\mathcal{G}_{d}(\tau, w) / \operatorname{vol}(\tau)\right)\right]$. For regularly sampled meshes, the geometric range should then be always a low value.

Mesh quality criteria. Geometric Modelling generally aims at generating meshes composed of wellshaped cells. The usual definition for well-shaped triangles are the aspect ratio $\mathcal{G}_{a}\left(\tau=\left(v_{1}, v_{2}\right), w\right)=$ $\frac{\left\|v_{1}-v_{2}\right\|^{2}+\left\|w-v_{1}\right\|^{2}+\left\|w-v_{2}\right\|^{2}}{\operatorname{area}\left(v_{1}, v_{2}, w\right)}$ and the Delaunay constraint $\mathcal{G}_{c}(\tau, w)=$ circumradius $(\tau \star w)$. The first one usually concerns rendering applications and for local mesh improvement [2], whereas the second one is widely used in reconstruction [6, 5, 10] and global remeshing [3, 4]. These criteria can also be used for the geometric function $\mathcal{R}$ with an exponential quantisation, noticing that they are bounded from below (by $\frac{16}{\sqrt{5}}$ for the aspect ration and $\frac{1}{2}$ for the circumradius normalised by the edge length (Figure 8). We can observe that the Delaunay criterion mimics the Ball Pivoting [6, 28] algorithm, where the geometric range encodes the variations of the ball radius. In higher dimension, these criteria can be extended using the Cayley-Menger determinant (especially for computing the circumradius [7]) or replaced by the cell volume for faster computation.
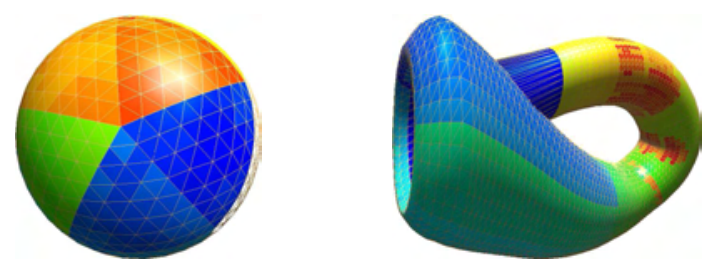

Figure 9: Traversal of a sphere and of a Klein bottle models, from cold to hot colours: good orders can improve the compression.

Traversal strategy. The criterion $\mathcal{G}$ actually depends of which front cell is chosen at each step. Therefore, the order in which the complex is traversed has an influence on the compression ratio, as most of the advancing front (greedy) algorithms (Figure 9). Normalised criteria as the distance or the circumradius will be a priori better quantised if the normalisation is bigger. Since the volume of the front cell gives is the natural normalisation (Figure 8), we store the front as a priority queue ordered by the volume of the cells. 


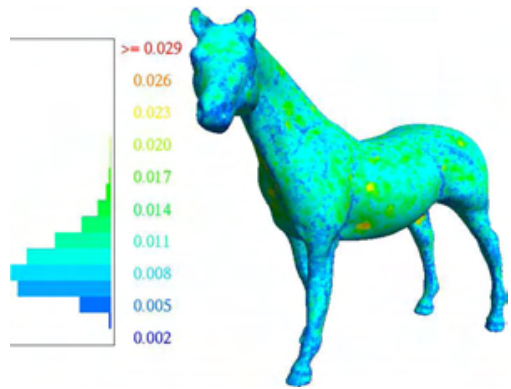

8(a): circumradius

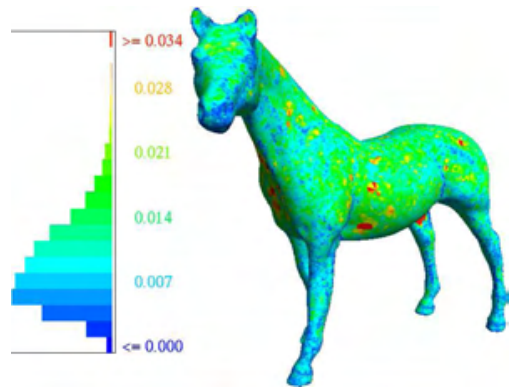

8(b): edge length

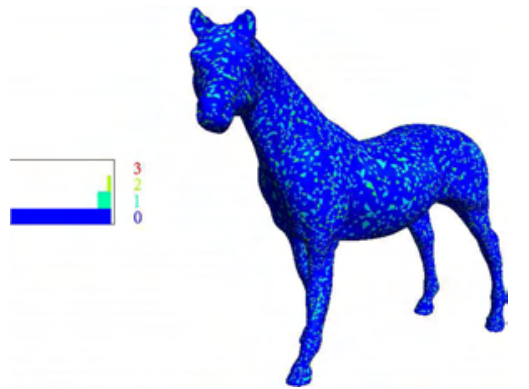

8(c): quantised range

Figure 8: Normalisation of the geometric range significantly reduces the entropy.

\section{Results}

Compression results. GEncode originally intended to compress better meshes that have a nice geometry. The Delaunay criterion is particularly adapted to reconstruction algorithms [6, 5, 10] or some techniques of re-meshing [3, 4]. The results of Tab. 1 shows this behaviour stands in practise, as the remeshed models and the scans sculptures are better compressed than the other models (Figure 10). Although the results presented here are of dimensions two and three, the algorithm has been implemented for any dimension and co-dimension, and the illustrations of this work represent the models decoded by our implementation. The only two features that were not implemented in high dimension are the non-pure compression and the manifoldness recognition (which is NP-hard).

Geometric priors. The adaptation power of GEncode allows using different priors on the same model to check which is the most efficient. Tab. 2 compares different methods on the same range of models as Tab.1, showing the percentage of best results for each quantised range, range precision and geometric criterion. The range precision represents the above-mentioned trade-off between range quan-

\begin{tabular}{l|rr} 
& Geometry & Connectivity \\
\hline animal & 19.343 & 1.980 \\
art & 19.561 & 1.491 \\
cad & 18.566 & 1.682 \\
math & 21.499 & 1.996 \\
medical & 21.220 & 2.411 \\
scans & 18.639 & 1.372 \\
\hline original & 19.334 & 2.246 \\
re-meshed & 18.882 & 1.269 \\
\hline all & 19.089 & 1.717
\end{tabular}

Table 1: GEncode compression ratio, in bits per vertex, using the Delaunay constraint for both the quantised range and the geometric criterion. These results are an average over 200 models, using an order one arithmetic coder. tisation and apex identifiers. The best result can be reached by various geometric criteria, particularly when the mesh is very regular or when the quantised range encodes almost completely the apex.

Comparison. GEncode compares nicely to existing compression scheme, although it is able to compress a wider range of models. Tab. 3 details some comparisons with the Edgebreaker algorithm [29, 27, 23] for the meshes illustrating this work. These comparisons were made using the parallelogram prediction for the Edgebreaker, with the same quantisation for the vertices (12 bits per coordinate). Observe that even for surfaces, connectivity-driven compression schemes handle with difficulty non-simple topology, as for the mechanical piece, which has a pinched vertex or the Klein bottle, which is not orientable, whereas these are handled gracefully by the GEncode.

GEncode turns out to be very effective for volumes embedded in $\mathbb{R}^{3}$ (Figure 11), as shown on Tab. 4 We compared with the connectivity-driven compression of [32] for the connectivity, and with the streaming compression of [16]. Although this last method shows some similarity with this work, the compression ratios of GEncode

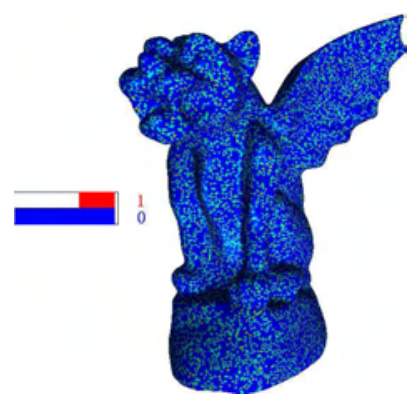

10(a): geometric range

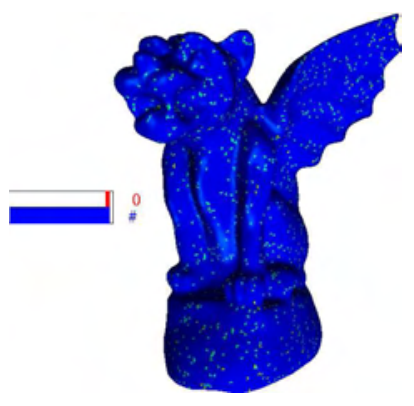

10(b): apex identifier
Figure 10: Compression of scanned models: the connectivity is encoded almost at zero rate: \# is not transmitted, and thus almost only 0 codes are encoded. 


\begin{tabular}{l|rr|rrr|rrrr} 
& \multicolumn{2}{|c|}{ Quantised Range $\mathcal{R}$} & \multicolumn{2}{|c|}{ Quantisation precision } & \multicolumn{4}{c}{ Geometric Criterion $\mathcal{G}$} \\
& distance & circumradius & rough & regular & detailed & distance & volume & aspect & circumradius \\
\hline animal & $17 \%$ & $83 \%$ & $7 \%$ & $17 \%$ & $76 \%$ & $100 \%$ & $83 \%$ & $77 \%$ & $83 \%$ \\
art & $5 \%$ & $95 \%$ & $43 \%$ & $90 \%$ & $0 \%$ & $26 \%$ & $31 \%$ & $67 \%$ & $54 \%$ \\
cad & $45 \%$ & $55 \%$ & $96 \%$ & $3 \%$ & $1 \%$ & $47 \%$ & $19 \%$ & $7 \%$ & $50 \%$ \\
math & $67 \%$ & $33 \%$ & $55 \%$ & $21 \%$ & $24 \%$ & $54 \%$ & $5 \%$ & $22 \%$ & $35 \%$ \\
medical & $0 \%$ & $100 \%$ & $100 \%$ & $0 \%$ & $0 \%$ & $61 \%$ & $0 \%$ & $0 \%$ & $39 \%$ \\
sculpture & $78 \%$ & $22 \%$ & $21 \%$ & $41 \%$ & $38 \%$ & $80 \%$ & $0 \%$ & $1 \%$ & $19 \%$
\end{tabular}

Table 2: Percentage of best results obtained by each geometric function, range precision, geometric criterion on the models used for Tab. 1. The best result can be reached by various geometric criteria, leading to a sum over $100 \%$.

\begin{tabular}{l|r|rr|rr} 
& $\mathrm{nv}$ & \multicolumn{2}{|c|}{ EB } & \multicolumn{2}{c}{ GEncode } \\
& & geom & conn & geom & conn \\
\hline terrain & 16641 & 17.09 & 0.28 & 13.11 & 2.71 \\
mechanical & 71150 & $N A$ & $N A$ & 15.86 & 0.82 \\
david & 24988 & 25.80 & 2.71 & 16.99 & 1.63 \\
horse & 19851 & 24.86 & 3.01 & 18.22 & 0.85 \\
gargoyle & 30059 & 20.99 & 2.32 & 18.38 & 0.58 \\
bunny & 34834 & 17.05 & 2.18 & 18.77 & 0.97 \\
blech & 4102 & 21.55 & 2.10 & 20.73 & 2.79 \\
fandisk & 6475 & 19.56 & 2.25 & 21.52 & 1.31 \\
klein & 4120 & $N A$ & $N A$ & 22.11 & 2.63 \\
sphere & 642 & 27.98 & 2.27 & 24.65 & 0.03 \\
rotor & 600 & 31.67 & 3.69 & 24.08 & 5.18
\end{tabular}

Table 3: GEncode compression ratio (in bits per vertex) for triangulated surfaces compared with Edgebreaker [29, 23]. The mechanical model is not manifold, and the Klein bottle is not orientable, which prevented the Edgebreaker to work.

are in average more than $35 \%$ better than for streaming compression, to the cost of being slower.

\section{Conclusion}

This works proposed a new geometry-driven compression scheme that is, to our knowledge, the first compression method that encodes meshes of any dimension and arbitrary topology, while being efficient compared to compression methods for triangulates surfaces. Prior knowledge on the model is used independently by GEncode: as an input for the geometric priors and as optimisation of the execution and compression ratio for the topological priors. In particular for scanned and re-meshed models, classical geometric priors such as the aspect ratio or Delaunay constraint improve significantly the compression ratio.

There is a computational price for the gain in compression: the localisation procedure, which consumes the main part of the execution time, is still slow for general geometrical ranges. In particular when comparing with streaming compression methods, the $35 \%$ gain required a three or four times more time in our experiments. Moreover, the separate

\begin{tabular}{|c|c|c|c|c|c|}
\hline & nv & $\begin{array}{l}\mathrm{G} \& \mathrm{~F} \\
\text { conn }\end{array}$ & \begin{tabular}{c}
\multicolumn{2}{c}{ Stream } \\
geom conn
\end{tabular} & $\begin{array}{r}\mathrm{GEr} \\
\text { geom }\end{array}$ & $\begin{array}{l}\text { code } \\
\text { conn }\end{array}$ \\
\hline $\mathrm{sph}$ & 12229 & 76.36 & 34.0075 .87 & 22.50 & 12.76 \\
\hline iron & 6103 & 51.93 & $29.90 \quad 25.17$ & 18.17 & 10.45 \\
\hline molecule & 5853 & 54.00 & 34.5723 .51 & 21.77 & 19.82 \\
\hline tric & 4344 & 55.41 & 34.8425 .82 & 19.39 & 18.06 \\
\hline fault & 3403 & 63.50 & 36.1735 .15 & 22.57 & 11.97 \\
\hline $\operatorname{dog}$ & 3286 & 55.50 & $34.31 \quad 17.13$ & 20.13 & 16.86 \\
\hline fandisk & 3000 & 64.85 & 36.2318 .11 & 24.26 & 9.06 \\
\hline turb & 2953 & $N A$ & 33.9111 .80 & 22.95 & 14.71 \\
\hline ratt & 2514 & $N A$ & 33.8411 .40 & 22.48 & 13.12 \\
\hline soli & 1004 & 60.52 & 41.0415 .69 & 26.68 & 9.22 \\
\hline $\mathrm{sph}$ & 986 & 65.01 & 41.1918 .76 & 31.46 & 10.01 \\
\hline $\mathbb{S}^{2}$ & 770 & 43.56 & $33.14 \quad 8.76$ & 21.87 & 8.34 \\
\hline bended cube & 400 & 57.86 & 42.5215 .36 & 23.54 & 14.59 \\
\hline & 234 & 48.62 & $47.18 \quad 15.01$ & 26.18 & 10.92 \\
\hline & 141 & 53.22 & 53.0519 .29 & 29.77 & 11.98 \\
\hline sphere100 & 100 & 55.84 & 57.3619 .68 & 31.49 & 8.61 \\
\hline
\end{tabular}

Table 4: GEncode compression ratio (in bits per vertex) for tetrahedral meshes compared with Grow \& Fold [32] and streaming compression [16].

encoding of the geometry still limits the final compression ratio. On one hand, the geometric compression can be improved, and the synthesis proposed in this work is a first attempt in that direction. On the other hand, improvements based on mixed geometry/connectivity encoding are feasible with GEncode.

\section{References}

[1] P. Alliez and M. Desbrun Valence-driven connectivity encoding of 3D meshes In Computer Graphics Forum, pages 480-489, 2001.

[2] P. Alliez, M. Meyer and M. Desbrun, Interactive Geometry Remeshing. In Siggraph, pages 347-354. ACM, 2002.

[3] P. Alliez, D. Cohen-Steiner, O. Devillers, B. Levy and M. Desbrun Anisotropic polygonal remeshing. In 


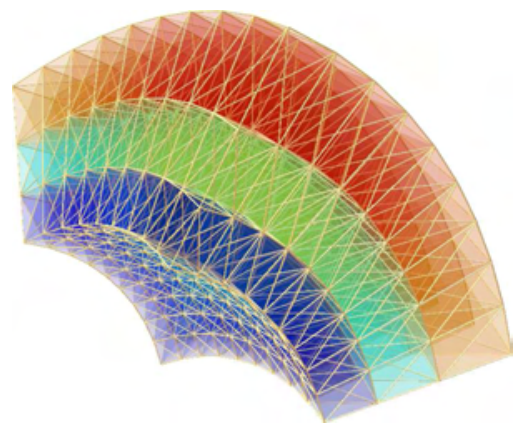

11(a): A bended cube, decompressed from cold to hot colours.

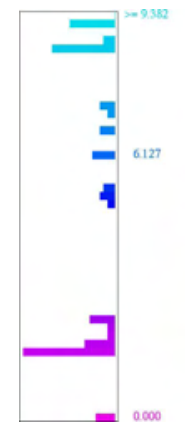

11(b): A mesh of poor quality, generated from points closed to the sphere $\mathbb{S}^{2}$, with the distribution of their circumradii.

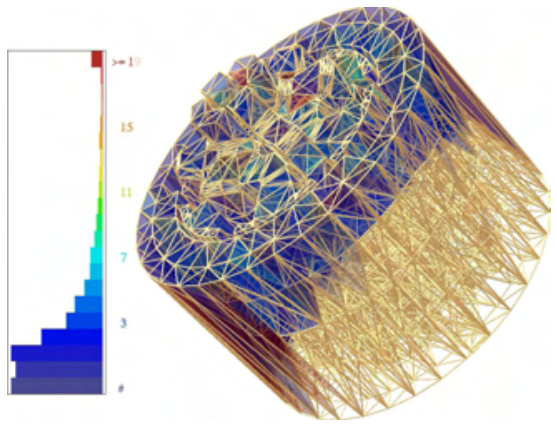

11(c): A turbine model, similar to the rotor surface, with the distribution of the apex identifiers during the decoding.

Figure 11: Compression on tetrahedral meshes works exactly as for triangulated surfaces.

Siggraph. ACM, 2003.

[4] P. Alliez, É. Colin de Verdière, O. Devillers and M. Isenburg. Isotropic surface remeshing. In Shape Modeling International. IEEE, 2003.

[5] N. Amenta, S. Choi and R. Kolluri The Power Crust, unions of balls, and the medial axis transform Computational Geometry: Theory and Applications, 19(23):127-153, 2001.

[6] F. Bernardini, J. Mittleman, H. Rushmeier, C. Silva and G. Taubin The Ball-Pivoting Algorithm for surface reconstruction. Transactions on Visualization and Computer Graphics, 5(4):349-359, 1999.

[7] L. M. Blumenthal Theory and applications of distance geometry. Chelsea, New York, 1970.

[8] M. Botsch, A. Wiratanaya and L. Kobbelt Efficient high quality rendering of point sampled geometry. In Eurographics Workshop on Rendering, pages 53-64, 2002.

[9] L. Castelli Aleardi and O. Devillers Canonical triangulation of a graph, with a coding application. INRIA preprint, 2004.

[10] D. Cohen-Steiner and T. K. F. Da A greedy Delaunay-based surface reconstruction algorithm. The Visual Computer, 20(1):4-16, 2002.

[11] V. Coors and J. Rossignac Delphi: geometry-based connectivity prediction in triangle mesh compression. The Visual Computer, 20(8-9):507-520, 2004.

[12] P.-M. Gandoin and O. Devillers, Progressive lossless compression of arbitrary simplicial complexes. In Siggraph, volume 21, pages 372-379. ACM, 2002.
[13] A. Guéziec, F. Bossen, G. Taubin and C. Silva, Efficient compression of non-manifold polygonal meshes. Computational Geometry: Theory and Applications, 14(1-3):137-166, 1999.

[14] S. Gumhold, S. Guthe and W. Strä̈er Tetrahedral mesh compression with the Cut-Border machine In Visualization, pages 51-58. IEEE, 1999.

[15] A. Hatcher Algebraic topology Cambridge University Press, 2002.

[16] M. Isenburg, P. Lindstrom, S. Gumhold and J. R. Shewchuk. Streaming Compression of Tetrahedral Volume Meshes, www.cs.unc.edu/ isenburg/research/sctvm.

[17] M. Isenburg and P. Alliez. Compressing hexahedral volume meshes. In Pacific Graphics, pages 284-293, 2002.

[18] F. Kälberer, K. Polthier, U. Reitebuch and M. Wardetzky. Freelence - coding with free valences. Computer Graphics Forum, 24(3):469-478, 2005.

[19] D. King and J. Rossignac. Guaranteed 3.67v bit encoding of planar triangle graphs. In Canadian Conference on Computational Geometry, pages 146-149, 1999.

[20] B. Kronrod and C. Gotsman Efficient coding of nontriangular mesh connectivity. Graphical Models, 63:263-275, 2001.

[21] H. Lee, P. Alliez and M. Desbrun, Angle-Analyzer: A Triangle-Quad Mesh Codec. In Eurographics, volume 21(3), 2002.

[22] H. Lee, M. Desbrun and P. Schröder. Progressive encoding of complex isosurfaces Transactions on Graphics, 22(3):471-476, 2003. 
[23] T. Lewiner, H. Lopes, J. Rossignac and A. W. Vieira Efficient Edgebreaker for surfaces of arbitrary topology In Sibgrapi, pages 218-225, Curitiba, Oct. 2004. IEEE.

[24] T. Lewiner, L. Velho, H. Lopes and V. Mello Simplicial isosurface compression. In Vision, Modeling and Visualization, pages 299-306, Stanford, 2004. IOS Press.

[25] T. Lewiner, M. Craizer, H. Lopes, S. Pesco, L. Velho and E. Medeiros, GEncode: geometry-driven compression in arbitrary dimension and co-dimension. In Sibgrapi, pages 249-256, Natal, Oct. 2005. IEEE.

[26] T. Lewiner Mesh Compression from Geometry. PhD thesis, Géométrica Project, INRIA-Sophia Antipolis, delivered by Université Paris VI, Dec. 2005. Advised by Jean-Daniel Boissonnat

[27] H. Lopes, J. Rossignac, A. Safonova, A. Szymczak and G. Tavares Edgebreaker: a simple compression for surfaces with handles In C. Hoffman and W. Bronsvort, editors, Solid Modeling and Applications, pages 289296, Saarbrücken, Germany, 2002. ACM.

[28] E. Medeiros, L. Velho and H. Lopes Restricted bpa: applying ball-pivoting on the plane. In Sibgrapi, pages 372-379, Curitiba, Oct. 2004. IEEE.

[29] J. Rossignac Edgebreaker: connectivity compression for triangle meshes. Transactions on Visualization and Computer Graphics, 5(1):47-61, 1999.

[30] J. Rossignac and D. Cardoze, Matchmaker: manifold BReps for non-manifold r-sets. In C. Hoffman and W. Bronsvort, editors, Solid Modeling and Applications, pages 31-41. ACM, 1999.

[31] C. E. Scheidegger, S. Fleishman and C. T. Silva, Triangulating point-set surfaces with bounded error. In Symposium on Geometry Processing, pages 63-72. Eurographics, 2005.

[32] A. Szymczak and J. Rossignac Grow \& Fold: compressing the connectivity of tetrahedral meshes Computer-Aided Design, 32(8/9):527-538, 2000.

[33] G. Taubin and J. Rossignac. Geometric compression through topological surgery. Transactions on Graphics, 17(2):84-115, 1998.

[34] G. Taubin Blic: bi-level isosurface compression. In Visualization, pages 451-458, Boston, Massachusetts, 2002. IEEE.

[35] C. Touma and C. Gotsman Triangle mesh compression. In Graphics Interface, pages 26-34, 1998. 